\title{
Mucolipidosis Type III: A Rare Disease in Differential Diagnosis of Joint Stiffness in Pediatric Rheumatology
}

\author{
Çiğdem Seher KASAPKARA, ${ }^{1}$ Meltem AKÇABOY, ${ }^{2}$ Fehime KARA EROĞLU, ${ }^{3}$ Betül Emine DERINNUUUU \\ ${ }^{1}$ Department of Pediatric Metabolism and Nutrition, Dr. Sami Ulus Maternity and Children's Health and \\ Diseases Research and Education Hospital, Ankara, Turkey \\ ${ }^{2}$ Department of Pediatrics, Dr. Sami Ulus Maternity and Children's Health and Diseases Research and Education Hospital, Ankara, Turkey \\ ${ }^{3}$ Department of Pediatric Nephrology, Dr. Sami Ulus Maternity and Children's Health and Diseases Research and Education Hospital, Ankara, Turkey \\ ${ }^{4}$ Department of Pediatric Radiology, Dr. Sami Ulus Maternity and Children's Health and Diseases Research and Education Hospital, Ankara, Turkey
}

\begin{abstract}
Mucolipidoses are metabolic disorders with autosomal recessive inheritance caused by deficiency of $\mathrm{N}$-acetylglucosamine- 1-phosphotransferase leading to accumulation of glycosaminoglycans and sphingolipids intracellularly. The differential diagnosis of mucolipidosis II or III is based on the age of onset, clinical findings and degree of severity. In this article, we present four pediatric patients with mucolipidosis III or pseudo-Hurler polydystrophy who admitted to our hospital with joint stiffness. They were from consanguineous families with characteristic radiographic findings. The joints were painless and the rheumatologic evaluation and inflammation markers were negative. Mucolipidosis is a rare disease in pediatric patients to remember in differential diagnosis of joint stiffness.

Keywords: Differential diagnosis; joint stiffness; mucolipidosis; pediatric rheumatology.
\end{abstract}

Mucolipidosis (ML) is a rare autosomal recessive inherited metabolic disorder of lysosomal metabolism characterized by defective processing of multiple lysosomal degradative enzymes due to the absent or deficient activity of N-acetylglucosamine-1-phosphotransferase. Deficient activity of $\mathrm{N}$-acetylglucosamine1-phosphotransferase leads to defective posttranslational modification of lysosomal enzymes, promoting the intracellular accumulation of both partly degraded glycosaminoglycans and sphingolipids. ${ }^{1-3}$ The trafficking process that transports the lysosomal enzymes to the interior of lysosomes is impaired. Thus the degradative enzymes accumulate at high concentrations in plasma serving as a diagnostic evidence for mucolipidosis II/III. The differential diagnosis of ML II or III is based on the age of onset, clinical findings and degree of severity. ${ }^{4,5}$ In this article, we present four pediatric patients with joint stiffness and diagnosed as ML III or pseudo-Hurler polydystrophy with characteristic radiographic findings to attract attention to this rare entity in pediatric rheumatology.

\section{CASE REPORT}

Case 1, 2- A five-and-a-half-year-old girl patient from Syrian origin referred to the Department of Pediatric Metabolism and Nutrition due to the presence of claw-hand deformity and joint stiffness (Figure 1). This girl was the second child of a consanguineous couple. Lateral cervical spine and posteroanterior chest radiographs of the patient diagnosed with ML III showed 


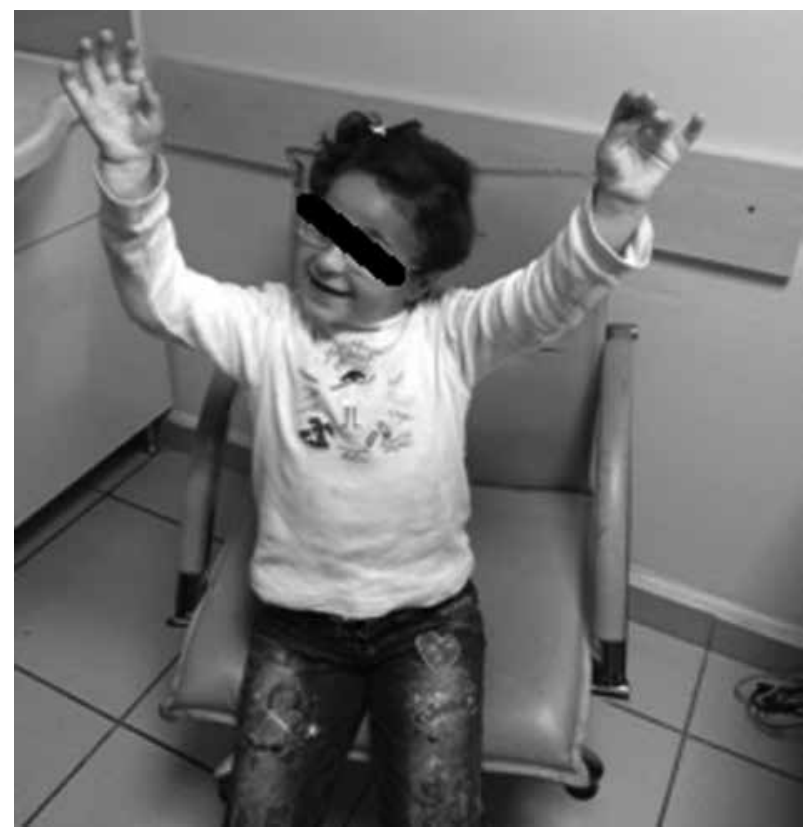

Figure 1. Physical appearance of case 1; Five-and-ahalf-year-old Syrian girl, showing short stature, claw-hand deformity, limited in maximum flexion and extension of the fingers, elbows and shoulders.

abnormal vertebral bodies with anterior vertebral beaking on the lateral cervical radiogram and chest X-ray demonstrated short and thick clavicles (Figure 2). In physical examination; her body weight was $15 \mathrm{~kg}$ (3\%-10\% percentile) and height was $106 \mathrm{~cm}$ (3\%-10\% percentile). Her neurological development was normal for her age. However, she had claw-hand deformity, limited in maximum flexion and extension of the fingers, elbows and shoulders. Ophthalmological and rheumatologic laboratory evaluations were normal, but cardiac examination revealed aortic insufficiency.

Her elder brother was eight years old and had more severe similar clinical findings. Around four years of age, skeletal alterations were manifested by short stature, difficulty to run, go upstairs and downstairs, joint stiffness and difficulty in leaning forward (Figure 3). His body weight was $22 \mathrm{~kg}$ (3\%-10\% percentile) and height was $120 \mathrm{~cm}$ (3\%-10\% percentile). He had mitral insufficiency. None of the patients had history of fever, extremity pain or swelling. Urine dermatan sulphate concentrations were elevated in both of them and the gross elevations of plasma betahexosaminidase, arylsulphatase $\mathrm{A}$ and alphamannosidase were consistent with a diagnosis of ML II/III. A written informed consent was obtained from the parents of patients.

Case 3, 4- A five-and-a-half-year-old girl patient from Turkish origin was admitted to the Department of Pediatric Metabolism and Nutrition due to the presence of claw-hand deformity (Figure 4). This girl was the sixth child of a consanguineous couple. Around three years of age, skeletal alterations were manifested by short stature, difficulty to run, go upstairs and downstairs and joint stiffness. In physical examination; her body weight was $19 \mathrm{~kg}$ (25\%-50\% percentile) and height was $111 \mathrm{~cm}$ (25\%-50\% percentile). Her neurological development was normal for her age. However, she had claw-hand deformity, limited in maximum flexion and extension of the fingers, elbows and shoulders. There was no history of fever, extremity pain, tenderness or swelling. Hand
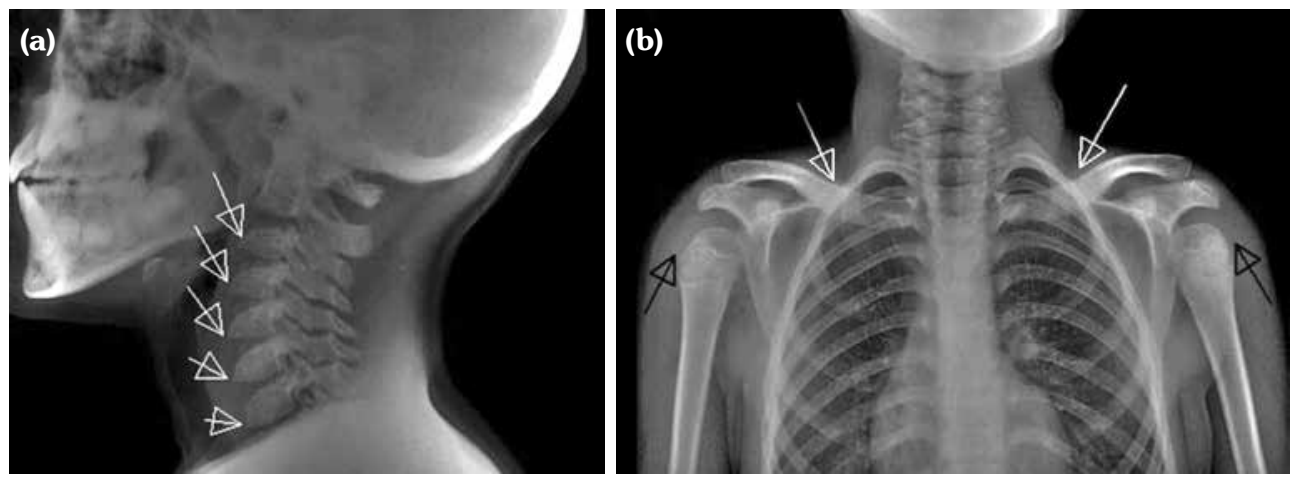

Figure 2. Lateral cervical spine and posteroanterior chest radiographs of case 1 diagnosed with mucolipidosis III. (a) On lateral cervical radiogram, note abnormal vertebral bodies with anterior vertebral beaking (white arrows). (b) Chest X-ray demonstrates short and thick clavicles (white arrows). Note slightly small humeral heads (black arrows). 


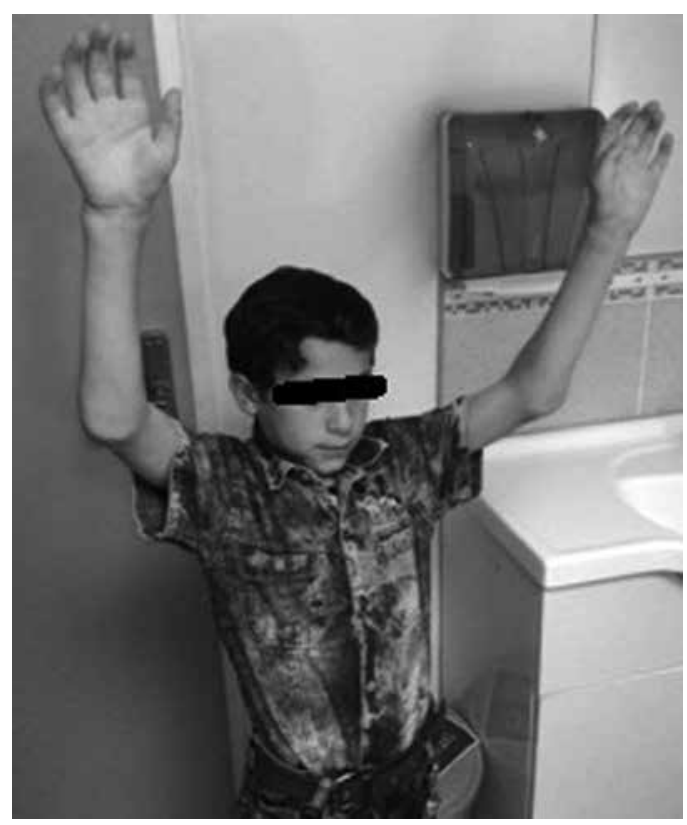

Figure 3. Physical appearance of case 2; Eightyear-old Syrian boy showing short stature, joint stiffness of elbows and shoulders.

radiograph showed short and wide metacarpal tubular bones. Pelvis radiograph demonstrated the flaring of the iliac wings, shallow acetabular fossae and lateral subluxation of the femoral heads (Figure 5). Ophthalmological and rheumatologic laboratory evaluations were normal, but cardiac

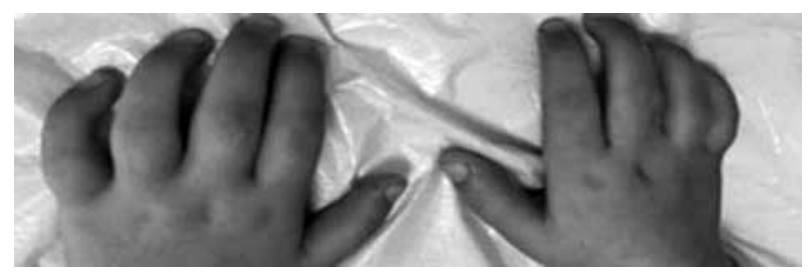

Figure 4. Claw-hand deformity (impossible flexion and maximum extension) in case 3 diagnosed with mucolipidosis III.

exam revealed aortic insufficiency and mitral valve prolapse. A written informed consent was obtained from the parents of the patient.

Her elder brother was 18 years old and had more severe similar clinical findings (Figure 6). His body weight was $41 \mathrm{~kg}(<3 \%$ percentile) and height was $147 \mathrm{~cm}(<3 \%$ percentile). $\mathrm{He}$ has scoliosis, dysostosis multiplex, mild mental retardation, waddling gait, height stunting and coarse face. Echocardiographic evaluation revealed aortic insufficiency. Urine dermatan sulphate concentrations were elevated in both of them and the gross elevations of plasma beta-hexosaminidase, arylsulphatase A and alpha-mannosidase were consistent with a diagnosis of ML II/III. A written informed consent was obtained from the parents of the patient.
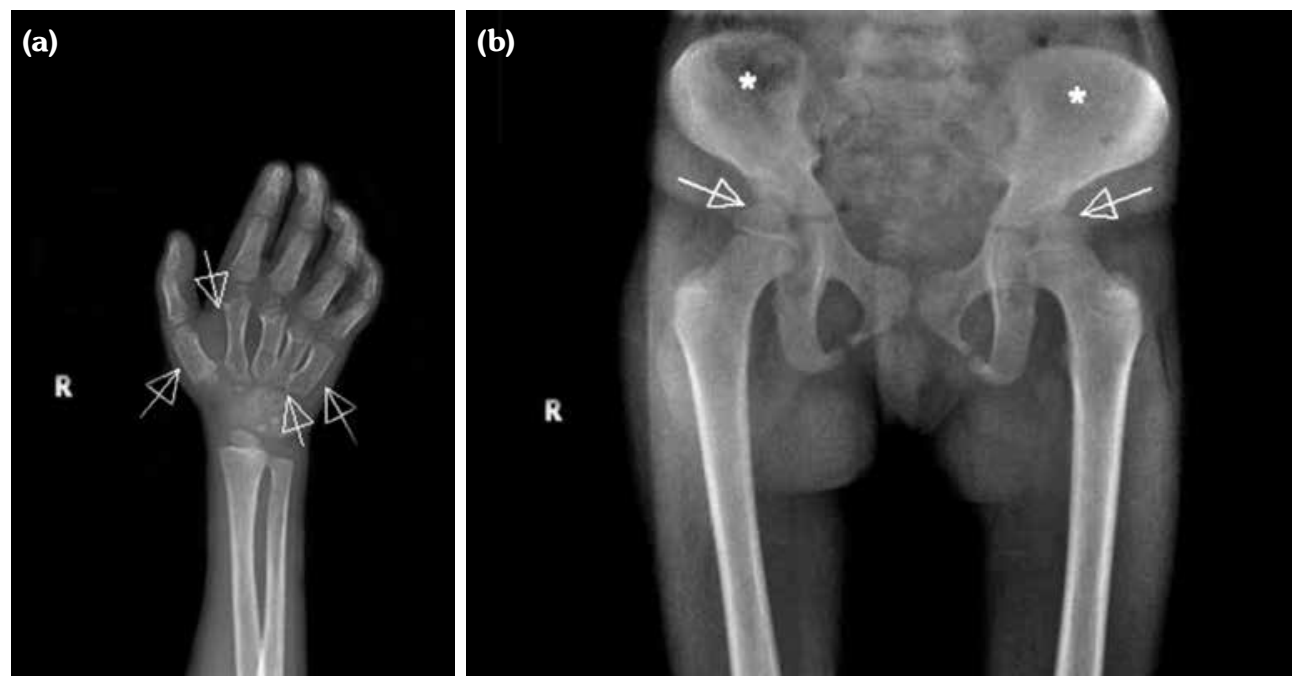

Figure 5. Hand and pelvis radiographs of case 3 diagnosed with mucolipidosis III. (a) Hand radiograph shows short and wide metacarpal tubular bones (white arrows). Also, note claw-hand deformity. (b) Pelvis radiograph demonstrates flaring of iliac wings (white asterisk), shallow acetabular fossae and lateral subluxation of femoral heads (white arrows). 


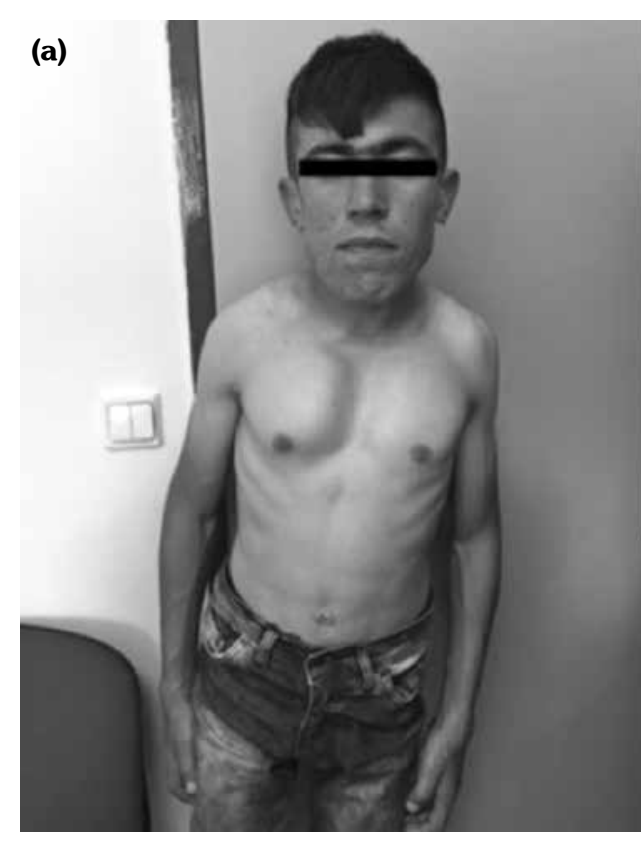

The demographic and clinical data, radiologic findings and laboratory evaluations of the patients are given in Table 1.

\section{DISCUSSION}

We reported four pediatric patients with complaints of immobility and painless joint stiffness and diagnosed as ML. ML is a rare autosomal recessive lysosomal storage disease, which can be classified into four types. ML II/III are caused by deficiency of the enzyme $\mathrm{N}$-acetylglucosamine- 1 phosphotransferase. ML III is a milder disorder with attenuated characteristics and survival to adulthood. Patients usually present between two and four years of age with symptoms referable to the joints. ${ }^{4-6}$ The typical clinical symptoms include short stature, cardiac valve involvement, normal intelligence or mild mental retardation, normal corneal appearance or steaminess of the cornea, and scoliosis and skeletal and orthopedic complications including hand and shoulder stiffness, claw-hand deformities, short iliac wings, erosion of the femoral heads, dysostosis multiplex of the vertebral bodies, long bones, skull, phalanges and clavicles with no to mild organomegaly. ${ }^{3-7}$ Cardiopulmonary complications are the usual reasons of mortality in patients with ML III. Aortic and mitral valve insufficiencies are

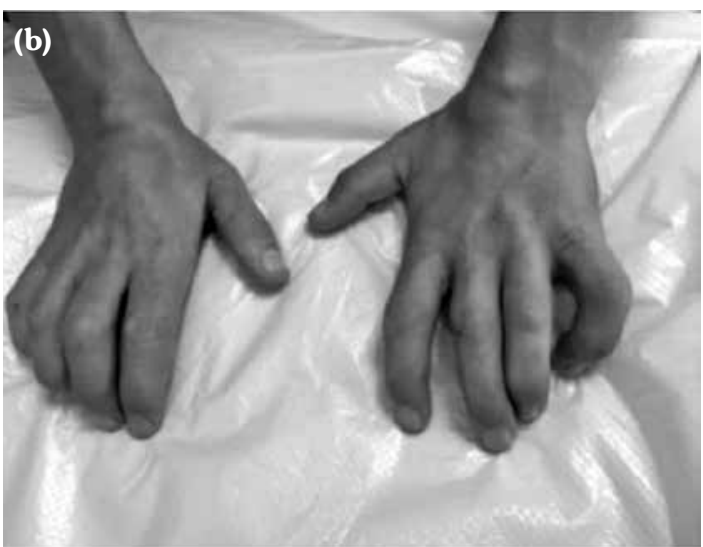

Figure 6. Physical appearance of Case 4; 18 years old Turkish male, (a) showing coarse face, short stature and scoliosis in physical examination, (b) hands of the patients showing contractures. the most common forms of cardiac involvement. ${ }^{1}$ All of our patients with ML III had cardiac valve insufficiency. A carpal tunnel syndrome may develop in these patients. Flexion contracture of knees, hips, and elbows may lead to shortness of stature. Progressive destructive changes in the hip may lead to a waddling gait. Late effects are destruction of the femoral heads and vertebral bodies leading to a compromised mobility. The laboratory findings of patients with ML III involve the abundance of some lysosomal enzymes in plasma including arylsulfatase. Genetic transmission is autosomal recessive and consanguinity was observed in all of our patients with ML III. ${ }^{8}$

The differential diagnoses of ML should include juvenile idiopathic rheumatoid arthritis, mucopolysaccharidoses, oligosaccharides and progressive pseudorheumatoid arthritis of childhood. ${ }^{6,7}$

The therapeutic methods for ML III are essentially symptomatic treatment and genetic counselling. Intravenous pamidronate has been used in order to reduce bone pain and improve mobility. Supportive orthopedic management and physiotherapy may be useful in such patients. In ML III, evolution is slow and patients can usually reach up to the fifth decade of life. Prenatal diagnosis is possible by assay of the enzyme in 


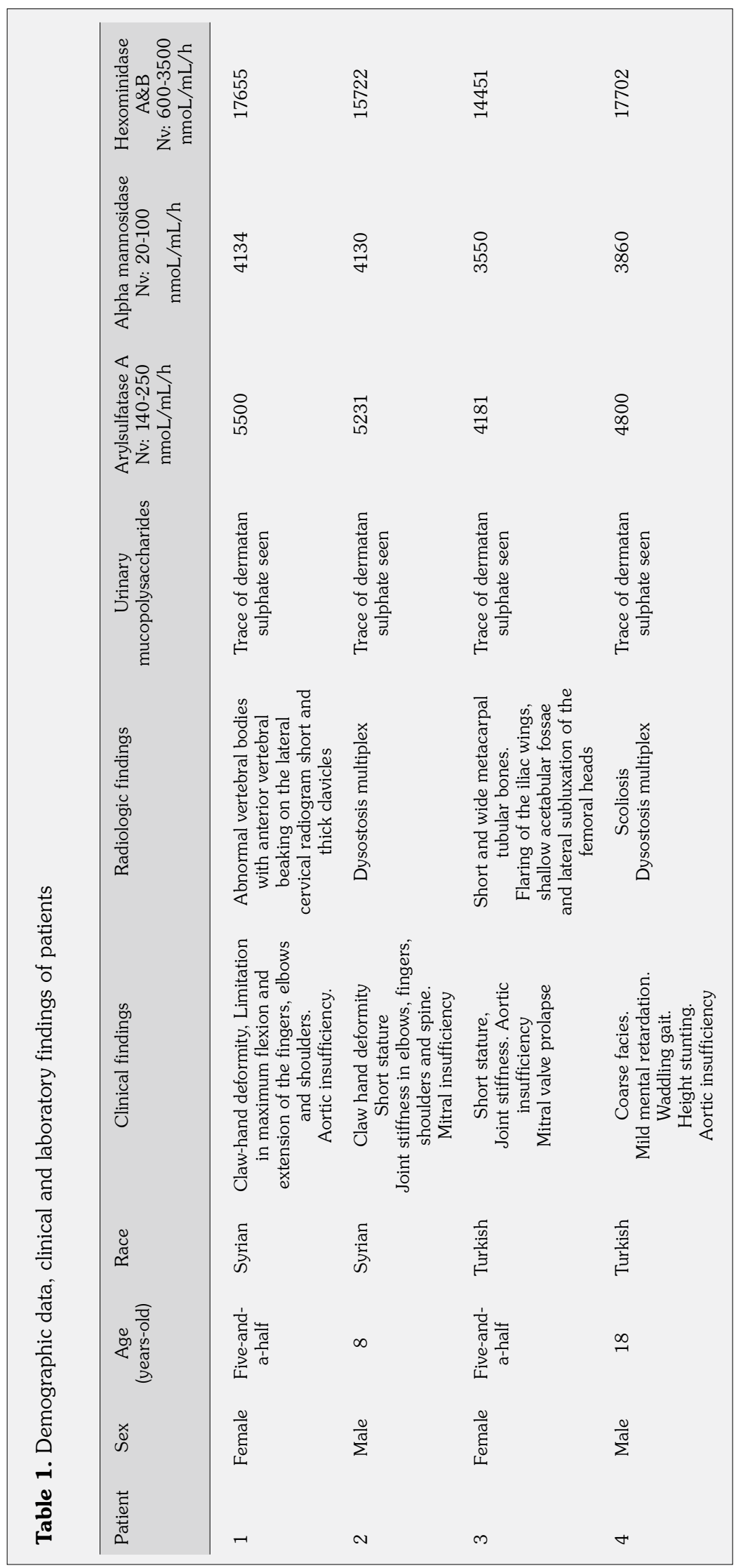


cultured amniocytes or chorionic villus cells or by molecular genetic analysis. ${ }^{5-8}$

Due to the manifestations in the skeletal system and involvement of joints in ML, the first application should be to the clinics of pediatric rheumatology. Family history, consanguinity, lack of pain and inflammation are key points to consider for the diagnosis of this rare disease.

\section{Declaration of conflicting interests}

The authors declared no conflicts of interest with respect to the authorship and/or publication of this article.

\section{Funding}

The authors received no financial support for the research and/or authorship of this article.

\section{REFERENCES}

1. Abualsuod A, Hacioglu Y, Vallurupalli S, Deshmukh A, Paydak H. Cardiac MRI findings in mucolipidosis
III. Acta Cardiol 2014;69:564-5.

2. Liu S, Zhang W, Shi H, Meng Y, Qiu Z. Three novel homozygous mutations in the GNPTG gene that cause mucolipidosis type III gamma. Gene 2014;535:294-8.

3. Pantoja Zarza L, Diez Morrondo C. Skeletal deformities in mucolipidosis III. Reumatol Clin 2014;10:340-1.

4. Hara M, Inokuchi T, Taniwaki T, Otomo T, Sakai N, Matsuishi T, et al. An adult patient with mucolipidosis III alpha/beta presenting with parkinsonism. Brain Dev 2013;35:462-5.

5. Khalifa H, Grubisa HS, Lee L, Lam EW. Enlarged follicles and temporomandibular joint abnormalities in mucolipidosis Type III. Dentomaxillofac Radiol 2013;42:22822014.

6. Simsek-Kiper PO, Topaloglu R, Sahin Y, Utine GE, Boduroglu K. Mucolipidosis type III in an adolescent presenting with atypical facial features and skeletal deformities. Genet Couns 2013;24:7-12.

7. Sharma R, Ramachandran S, Bhat D, Bindu PS, Devi BI. Bilateral carpal tunnel syndrome in two children: common manifestation of an uncommon disease. Pediatr Neurosurg 2013;49:300-2.

8. Cavalcante WC, Santos LC, Dos Santos JN, de Vasconcellos SJ, de Azevedo RA, Dos Santos JN. Oral findings in patients with mucolipidosis type III. Braz Dent J 2012;23:461-6. 